\title{
Disproof of the List Hadwiger Conjecture
}

\author{
János Barát* \\ School of Mathematical Sciences \\ Monash University \\ VIC 3800, Australia \\ janos.barat@monash.edu
}

\author{
Gwenaël Joret ${ }^{\dagger}$ \\ Département d'Informatique \\ Université Libre de Bruxelles \\ Brussels, Belgium \\ gjoret@ulb.ac.be
}

\author{
David R. Wood ${ }^{\ddagger}$ \\ Department of Mathematics and Statistics \\ The University of Melbourne \\ Melbourne, Australia \\ woodd@unimelb.edu.au
}

Submitted: Oct 11, 2011; Accepted: Dec 4, 2011; Published: Dec 12, 2011

Mathematics Subject Classifications: 05C83, 05C15

\begin{abstract}
The List Hadwiger Conjecture asserts that every $K_{t}$-minor-free graph is $t$ choosable. We disprove this conjecture by constructing a $K_{3 t+2}$-minor-free graph that is not $4 t$-choosable for every integer $t \geq 1$.
\end{abstract}

\section{Introduction}

In 1943, Hadwiger [6] made the following conjecture, which is widely considered to be one of the most important open problems in graph theory; see [28] for a survey ${ }^{1}$.

Hadwiger Conjecture. Every $K_{t}$-minor-free graph is $(t-1)$-colourable.

The Hadwiger Conjecture holds for $t \leq 6$ (see $[3,6,19,20,30])$ and is open for $t \geq 7$. In fact, the following more general conjecture is open.

\footnotetext{
${ }^{*}$ Research supported by OTKA Grant PD 75837 and and K 76099.

${ }^{\dagger}$ Postdoctoral Researcher of the Fonds National de la Recherche Scientifique (F.R.S.-FNRS). Supported in part by the Actions de Recherche Concertées (ARC) fund of the Communauté française de Belgique. Also supported by an Endeavour Fellowship from the Australian Government.

${ }^{\ddagger}$ Supported by a QEII Fellowship from the Australian Research Council.

${ }^{1}$ See $[2]$ for undefined graph-theoretic terminology. Let $[a, b]:=\{a, a+1, \ldots, b\}$.
} 
Weak Hadwiger Conjecture. Every $K_{t}$-minor-free graph is ct-colourable for some constant $c \geq 1$.

It is natural to consider analogous conjectures for list colourings ${ }^{2}$. First, consider the choosability of planar graphs. Erdős et al. [5] conjectured that some planar graph is not 4choosable, and that every planar graph is 5 -choosable. The first conjecture was verified by Voigt [29] and the second by Thomassen [27]. Incidentally, Borowiecki [1] asked whether every $K_{t}$-minor-free graph is $(t-1)$-choosable, which is true for $t \leq 4$ but false for $t=5$ by Voigt's example. The following natural conjecture arises (see [10,32], and see [34] for related conjectures).

List Hadwiger Conjecture. Every $K_{t}$-minor-free graph is t-choosable.

The List Hadwiger Conjecture holds for $t \leq 5$ (see [7, 22, 33]). Again the following more general conjecture, first stated by Kawarabayashi and Mohar [10], is open.

Weak List Hadwiger Conjecture. Every $K_{t}$-minor-free graph is ct-choosable for some constant $c \geq 1$.

In this paper we disprove the List Hadwiger Conjecture for $t \geq 8$, and prove that $c \geq \frac{4}{3}$ in the Weak List Hadwiger Conjecture.

Theorem 1. For every integer $t \geq 1$,

(a) there is a $K_{3 t+2}$-minor-free graph that is not $4 t$-choosable.

(b) there is a $K_{3 t+1}$-minor-free graph that is not $(4 t-2)$-choosable,

(c) there is a $K_{3 t}$-minor-free graph that is not $(4 t-3)$-choosable.

Before proving Theorem 1, note that adding a dominant vertex to a graph does not necessarily increase the choice number (as it does for the chromatic number). For example, $K_{3,3}$ is 3 -choosable but not 2-choosable. Adding one dominant vertex to $K_{3,3}$ gives $K_{1,3,3}$, which again is 3-choosable [18]. In fact, this property holds for infinitely many complete bipartite graphs [18]; also see [21]. On the other hand, a referee made the following observation which readily implies that parts (b) and (c) of Theorem 1 are consequences of part (a). That said, parts (b) and (c) can also be proved using the same method as that used to prove part (a), and that is what we do.

Proposition 2. If $G$ is a $K_{t}$-minor-free graph that is not $k$-choosable, then there is a $K_{t+1}$-minor-free graph that is not $(k+1)$-choosable.

\footnotetext{
${ }^{2} \mathrm{~A}$ list-assignment of a graph $G$ is a function $L$ that assigns to each vertex $v$ of $G$ a set $L(v)$ of colours. $G$ is $L$-colourable if there is a colouring of $G$ such that the colour assigned to each vertex $v$ is in $L(v)$. $G$ is $k$-choosable if $G$ is $L$-colourable for every list-assignment $L$ with $|L(v)| \geq k$ for each vertex $v$ of $G$. The choice number of $G$ is the minimum integer $k$ such that $G$ is $k$-choosable. If $G$ is $k$-choosable then $G$ is also $k$-colourable - just use the same set of $k$ colours for each vertex. Thus the choice number of $G$ is at least the chromatic number of $G$. See [34] for a survey on list colouring.
} 
Proof. Let $L$ be a list-assignment that proves that $G$ is not $k$-choosable. Assume that no integer in $[1, k+1]$ appears in the lists of $L$. For $i \in[1, k+1]$, let $G_{i}^{\prime}$ be a copy of $G$ with list-assignment $L^{\prime}(v):=L(v) \cup\{i\}$ for each vertex $v$ of $G_{i}^{\prime}$. In every $L^{\prime}$-colouring of $G_{i}^{\prime}$ some vertex is coloured $i$. Let $G^{\prime}$ be the graph obtained from the disjoint union of $G_{1}^{\prime}, \ldots, G_{k+1}^{\prime}$ by adding a dominant vertex $u$. Let $L^{\prime}(u):=[1, k+1]$. Clearly $G^{\prime}$ is $K_{t+1}$-minor-free. Say $G^{\prime}$ is $L^{\prime}$-colourable, and $u$ is coloured $i \in L^{\prime}(u)=[1, k+1]$. Then no vertex in $G_{i}^{\prime}$ is coloured $i$, which is a contradiction. Hence $G^{\prime}$ is not $L^{\prime}$-colourable, and $G^{\prime}$ is not $(k+1)$-choosable, as desired.

\section{Proof of Theorem 1}

Let $G_{1}$ and $G_{2}$ be graphs, and let $S_{i}$ be a $k$-clique in each $G_{i}$. Let $G$ be a graph obtained from the disjoint union of $G_{1}$ and $G_{2}$ by pairing the vertices in $S_{1}$ and $S_{2}$ and identifying each pair. Then $G$ is said to be obtained by pasting $G_{1}$ and $G_{2}$ on $S_{1}$ and $S_{2}$. The following lemma is well known.

Lemma 3. Let $G_{1}$ and $G_{2}$ be $K_{t}$-minor-free graphs. Let $S_{i}$ be a k-clique in each $G_{i}$. Let $G$ be a pasting of $G_{1}$ and $G_{2}$ on $S_{1}$ and $S_{2}$. Then $G$ is $K_{t}$-minor-free.

Proof. Suppose on the contrary that $K_{t+1}$ is a minor of $G$. Let $X_{1}, \ldots, X_{t+1}$ be the corresponding branch sets. If some $X_{i}$ does not intersect $G_{1}$ and some $X_{j}$ does not intersect $G_{2}$, then no edge joins $X_{i}$ and $X_{j}$, which is a contradiction. Thus, without loss of generality, each $X_{i}$ intersects $G_{1}$. Let $X_{i}^{\prime}:=G_{1}\left[X_{i}\right]$. Since $S_{1}$ is a clique, $X_{i}^{\prime}$ is connected. Thus $X_{1}^{\prime}, \ldots, X_{t+1}^{\prime}$ are the branch sets of a $K_{t+1}$-minor in $G_{1}$. This contradiction proves that $G$ is $K_{t}$-minor-free.

Let $K_{r \times 2}$ be the complete $r$-partite graph with $r$ colour classes of size 2 . Let $K_{1, r \times 2}$ be the complete $(r+1)$-partite graph with $r$ colour classes of size 2 and one colour class of size 1 . That is, $K_{r \times 2}$ and $K_{1, r \times 2}$ are respectively obtained from $K_{2 r}$ and $K_{2 r+1}$ by deleting a matching of $r$ edges. The following lemma will be useful.

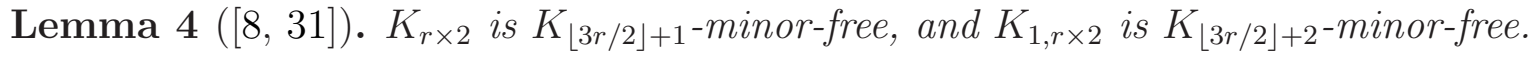

Proof of Theorem 1. Our goal is to construct a $K_{p}$-minor-free graph and a non-achievable list assignment with $q$ colours per vertex, where the integers $p, q$ and $r$ and a graph $H$ are defined in the following table. Let $\left\{v_{1} w_{1}, \ldots, v_{r} w_{r}\right\}$ be the deleted matching in $H$. By Lemma 4, the calculation in the table shows that $H$ is $K_{p}$-minor-free.

\begin{tabular}{c|ccccc}
\hline case & $p$ & $q$ & $r$ & $H$ & \\
\hline (a) & $3 t+2$ & $4 t$ & $2 t+1$ & $K_{r \times 2}$ & $\left\lfloor\frac{3}{2} r\right\rfloor+1=3 t+2=p$ \\
(b) & $3 t+1$ & $4 t-2$ & $2 t$ & $K_{r \times 2}$ & $\left\lfloor\frac{3}{2} r\right\rfloor+1=3 t+1=p$ \\
(c) & $3 t$ & $4 t-3$ & $2 t-1$ & $K_{1, r \times 2}$ & $\left\lfloor\frac{3}{2} r\right\rfloor+2=3 t=p$ \\
\hline
\end{tabular}

For each vector $\left(c_{1}, \ldots, c_{r}\right) \in[1, q]^{r}$, let $H\left(c_{1}, \ldots, c_{r}\right)$ be a copy of $H$ with the following list assignment. For each $i \in[1, r]$, let $L\left(w_{i}\right):=[1, q+1] \backslash\left\{c_{i}\right\}$. Let $L(u):=[1, q]$ for 
each remaining vertex $u$. There are $q+1$ colours in total, and $|V(H)|=q+2$. Thus in every $L$-colouring of $H$, two non-adjacent vertices receive the same colour. That is, $\operatorname{col}\left(v_{i}\right)=\operatorname{col}\left(w_{i}\right)$ for some $i \in[1, r]$. Since each $c_{i} \notin L\left(w_{i}\right)$, it is not the case that each vertex $v_{i}$ is coloured $c_{i}$.

Let $G$ be the graph obtained by pasting all the graphs $H\left(c_{1}, \ldots, c_{r}\right)$, where $\left(c_{1}, \ldots, c_{r}\right) \in[1, q]^{r}$, on the clique $\left\{v_{1}, \ldots, v_{r}\right\}$. The list assignment $L$ is well defined for $G$ since $L\left(v_{i}\right)=[1, q]$. By Lemma $3, G$ is $K_{p}$-minor-free. Suppose that $G$ is $L$-colourable. Let $c_{i}$ be the colour assigned to each vertex $v_{i}$. Thus $c_{i} \in L\left(v_{i}\right)=[1, q]$. Hence, as proved above, the copy $H\left(c_{1}, \ldots, c_{r}\right)$ is not $L$-colourable. This contradiction proves that $G$ is not $L$-colourable. Each vertex of $G$ has a list of $q$ colours in $L$. Therefore $G$ is not $q$-choosable. (It is easily seen that $G$ is $q$-degenerate ${ }^{3}$, implying $G$ is $(q+1)$-choosable.)

Note that this proof was inspired by the construction of a non-4-choosable planar graph by Mirzakhani [17].

\section{Conclusion}

Theorem 1 disproves the List Hadwiger Conjecture. However, list colourings remain a viable approach for attacking Hadwiger's Conjecture. Indeed, list colourings provide potential routes around some of the known obstacles, such as large minimum degree, and lack of exact structure theorems; see [10, 13, 32, 33].

The following table gives the best known lower and upper bounds on the maximum choice number of $K_{t}$-minor-free graphs. Each lower bound is a special case of Theorem 1 . Each upper bound (except $t=5$ ) follows from the following degeneracy results. Every $K_{3}$-minor-free graph (that is, every forest) is 1-degenerate. Dirac [4] proved that every $K_{4}$-minor-free graph is 2-degenerate. Mader [16] proved that for $t \leq 7$, every $K_{t}$-minorfree graph is $(2 t-5)$-degenerate. Jørgensen [9] and Song and Thomas [23] proved the same result for $t=8$ and $t=9$ respectively. Song [24] proved that every $K_{10}$-minor-free graph is 21 -degenerate, and that every $K_{11}$-minor-free graph is 25 -degenerate. In general, Kostochka $[14,15]$ and Thomason $[25,26]$ independently proved that every $K_{t}$-minor-free graph is $\mathcal{O}(t \sqrt{\log t})$-degenerate.

\begin{tabular}{c|ccccccccccc}
\hline$t$ & 3 & 4 & 5 & 6 & 7 & 8 & 9 & 10 & 11 & $\cdots$ & $t$ \\
\hline lower bound & 2 & 3 & 5 & 6 & 7 & 9 & 10 & 11 & 13 & $\cdots$ & $\frac{4}{3} t-c$ \\
upper bound & 2 & 3 & 5 & 8 & 10 & 12 & 14 & 22 & 26 & $\cdots$ & $\mathcal{O}(t \sqrt{\log t})$ \\
\hline
\end{tabular}

The following natural open problem arises: Is every $K_{6}$-minor-free graph 6 -choosable? It is even open whether every $K_{6}$-minor-free graph is 7 -choosable. This would be implied if every $K_{6}$-minor-free graph is 6 -degenerate (and we conjecture that this is true). Equivalently, we conjecture that every graph with minimum degree at least 7 contain a $K_{6}$-minor.

\footnotetext{
${ }^{3} \mathrm{~A}$ graph is $d$-degenerate if every subgraph has minimum degree at most $d$. Clearly every $d$-degenerate graph is $(d+1)$-choosable.
} 
It is even open whether every 7 -connected graph contains a $K_{6}$-minor. This would be implied by Jørgensen's conjecture, which asserts that every 6 -connected $K_{6}$-minor-free graph is apex ${ }^{4}$. Jørgensen's conjecture was recently proved for sufficiently large graphs $[11,12]$.

Acknowledgements: Thanks to Louis Esperet for stimulating discussions, and thanks to a referee for suggesting Proposition 2.

\section{References}

[1] Mieczyslaw Borowiecki. Research problem 172. Discrete Math., 121:235-236, 1993. doi: 10.1016/0012-365X(93)90557-A.

[2] Reinhard Diestel. Graph theory, vol. 173 of Graduate Texts in Mathematics. Springer, 4th edn., 2010. http://diestel-graph-theory.com/. MR: 2744811.

[3] Gabriel A. Dirac. A property of 4-chromatic graphs and some remarks on critical graphs. J. London Math. Soc., 27:85-92, 1952. doi:10.1112/jlms/s1-27.1.85. MR: 0045371.

[4] Gabriel A. Dirac. Homomorphism theorems for graphs. Math. Ann., 153:69-80, 1964. doi: 10.1007/BF01361708. MR: 0160203.

[5] Paul Erdős, Arthur L. Rubin, and Herbert Taylor. Choosability in graphs. In Proc. West Coast Conference on Combinatorics, Graph Theory and Computing, vol. XXVI of Congress. Numer., pp. 125-157. Utilitas Math., 1980. http://www.renyi.hu/ p_erdos/1980-07.pdf. MR:593902.

[6] Hugo Hadwiger. Über eine Klassifikation der Streckenkomplexe. Vierteljschr. Naturforsch. Ges. Zürich, 88:133-142, 1943. MR:0012237.

[7] Wenjie He, Wenjing Miao, and Yufa Shen. Another proof of the 5choosability of $K_{5}$-minor-free graphs. Discrete Math., 308(17):4024-4026, 2008. doi: 10.1016/j.disc.2007.07.089. MR: 2418108 .

[8] Jaroslav Ivančo. Some results on the Hadwiger numbers of graphs. Math. Slovaca, 38(3):221-226, 1988. MR: 977899.

[9] Leif K. Jørgensen. Contractions to $K_{8}$. J. Graph Theory, 18(5):431-448, 1994. doi: 10.1002/jgt.3190180502. MR: 1283309.

[10] Ken-IChi Kawarabayashi And Bojan Mohar. A relaxed Hadwiger's conjecture for list colorings. J. Combin. Theory Ser. B, 97(4):647-651, 2007. doi: 10.1016/j.jctb.2006.11.002. MR: 2325803.

\footnotetext{
${ }^{4} \mathrm{~A}$ graph $G$ is apex if $G-v$ is planar for some vertex $v \in V(G)$.
} 
[11] Ken-ichi Kawarabayashi, Serguei Norine, Robin Thomas, and Paul WolLAN. $K_{6}$ minors in 6-connected graphs of bounded tree-width. 2008.

[12] Ken-ichi Kawarabayashi, Serguei Norine, Robin Thomas, and Paul WolLAN. $K_{6}$ minors in large 6-connected graphs. 2008.

[13] Ken-ichi Kawarabayashi and Bruce Reed. Hadwiger's conjecture is decidable. In Proc. 41st Annual ACM Symposium on Theory of Computing (STOC '09), pp. 445-454. ACM, 2009. doi: 10.1145/1536414.1536476.

[14] Alexandr V. Kostochka. The minimum Hadwiger number for graphs with a given mean degree of vertices. Metody Diskret. Analiz., 38:37-58, 1982. MR:0713722.

[15] Alexandr V. Kostochka. Lower bound of the Hadwiger number of graphs by their average degree. Combinatorica, 4(4):307-316, 1984. doi: 10.1007/BF02579141. MR: 0779891.

[16] Wolfgang Mader. Homomorphiesätze für Graphen. Math. Ann., 178:154-168, 1968. doi: 10.1007/BF01350657. MR: 0229550.

[17] Maryam Mirzakhani. A small non-4-choosable planar graph. Bull. Inst. Combin. Appl., 17:15-18, 1996. MR: 1386951.

[18] Kyoji Oнва. Choice number of complete multipartite graphs with part size at most three. Ars Combin., 72:133-139, 2004. MR: 2069052.

[19] Neil Robertson, Daniel P. Sanders, Paul D. Seymour, and Robin Thomas. The four-colour theorem. J. Combin. Theory Ser. B, 70(1):2-44, 1997. doi: 10.1006/jctb.1997.1750. MR: 1441258.

[20] Neil Robertson, Paul D. Seymour, and Robin Thomas. Hadwiger's conjecture for $K_{6}$-free graphs. Combinatorica, 13(3):279-361, 1993. doi: 10.1007/BF01202354. MR: 1238823.

[21] Yufa Shen, Wenjie He, Guoping Zheng, Yanning Wang, and Lingmin ZHANG. On choosability of some complete multipartite graphs and Ohba's conjecture. Discrete Math., 308(1):136-143, 2008. doi:10.1016/j.disc.2007.03.059. MR:2370527.

[22] Riste Škrekovski. Choosability of $K_{5}$-minor-free graphs. Discrete Math., $190(1-$ 3):223-226, 1998. doi: 10.1016/S0012-365X(98)00158-7. MR: 1639710.

[23] Zi-Xia Song And Robin Thomas. The extremal function for $K_{9}$ minors. J. Combin. Theory Ser. B, 96(2):240-252, 2006. doi:10.1016/j.jctb.2005.07.008. MR: 2208353.

[24] Zixia Song. Extremal Functions for Contractions of Graphs. Ph.D. thesis, Georgia Institute of Technology, USA, 2004. CiteSeer: 10.1.1.92.1275. MR: 2706190. 
[25] Andrew Thomason. An extremal function for contractions of graphs. Math. Proc. Cambridge Philos. Soc., 95(2):261-265, 1984. doi: 10.1017/S0305004100061521. MR: 0735367.

[26] Andrew Thomason. The extremal function for complete minors. J. Combin. Theory Ser. B, 81(2):318-338, 2001. doi:10.1006/jctb.2000.2013. MR: 1814910.

[27] Carsten Thomassen. Every planar graph is 5-choosable. J. Combin. Theory Ser. B, 62(1):180-181, 1994. doi: 10.1006/jctb.1994.1062. MR: 1290638 .

[28] Bjarne Toft. A survey of Hadwiger's conjecture. Congr. Numer., 115:249-283, 1996. MR: 1411244.

[29] Margit Voigt. List colourings of planar graphs. Discrete Math., 120(1-3):215-219, 1993. doi: 10.1016/0012-365X(93)90579-I. MR: 1235909.

[30] Klaus Wagner. Über eine Eigenschaft der ebene Komplexe. Math. Ann., 114:570590, 1937. doi: 10.1007/BF01594196. MR: 1513158.

[31] David R. Wood. On the maximum number of cliques in a graph. Graphs Combin., 23(3):337-352, 2007. doi: 10.1007/s00373-007-0738-8. MR: 2320588.

[32] David R. Wood. Contractibility and the Hadwiger conjecture. European J. Combin., 31(8):2102-2109, 2010. doi: 10.1016/j.ejc.2010.05.015. MR: 2718284.

[33] David R. Wood and Svante Linusson. Thomassen's choosability argument revisited. SIAM J. Discrete Math., 24(4):1632-1637, 2010. doi: 10.1137/100796649. MR: 2746713 .

[34] Douglas R. Woodall. List colourings of graphs. In Surveys in combinatorics, vol. 288 of London Math. Soc. Lecture Note Ser., pp. 269-301. Cambridge Univ. Press, 2001. MR: 1850711. 